\title{
Intercalated Treatment Following Rebiopsy Is Associated with a Shorter Progression-Free Survival of Osimertinib Treatment
}

\author{
Jeng-Sen Tseng, MD, $P h D^{1,2}$ \\ Tsung-Ying Yang, $\mathrm{MD}, \mathrm{PhD}^{1,2}$ \\ Kun-Chieh Chen, MD, PhD' \\ Kuo-Hsuan Hsu, MD,3,4 \\ Yen-Hsiang Huang, MD' \\ Kang-Yi Su, PhD $D^{5,6}$ \\ Sung-Liang $Y u, P h D^{5,6,7,9,9}$ \\ Gee-Chen Chang, MD, PhD',2,10
}

\begin{abstract}
${ }^{1}$ Division of Chest Medicine, Department of Internal Medicine, Taichung Veterans General Hospital, Taichung, ${ }^{2}$ Faculty of Medicine, School of Medicine, National Yang-Ming University, Taipei, ${ }^{3}$ Institute of Biomedical Sciences, National Chung Hsing University, Taichung, ${ }^{4}$ Division of Critical Care and Respiratory Therapy, Department of Internal Medicine, Taichung Veterans General Hospital, Taichung, ${ }^{5}$ Department of Clinical Laboratory Sciences and Medical Biotechnology, College of Medicine, National Taiwan University, Taipei, ${ }^{6}$ Department of Laboratory Medicine, National Taiwan University Hospital, Taipei, ${ }^{7}$ Center of Genomic Medicine, National Taiwan University College of Medicine, Taipei, ${ }^{8}$ Department of Pathology and Graduate Institute of Pathology, National Taiwan University College of Medicine, Taipei, ${ }^{9}$ Center for Optoelectronic Biomedicine, National Taiwan University College of Medicine, Taipei, ${ }^{10}$ Comprehensive Cancer Center, Taichung Veterans General Hospital, Taichung, Taiwan
\end{abstract}

Correspondence: Gee-Chen Chang, MD, PhD Division of Chest Medicine, Department of Internal Medicine, Taichung Veterans General Hospital, No. 1650, Sect. 4, Taiwan Boulevard, Taichung, 407 Taiwan, R.O.C.

Tel: 886-4-23592525 (ext. 3200)

Fax: 886-4-23552590

E-mail: august@vghtc.gov.tw

Received September 28, 2017

Accepted December 8, 2017

Published Online December 11, 2017

\section{Purpose}

Epidermal growth factor receptor (EGFR) T790M mutation serves as an important predictor of osimertinib efficacy. However, little is known about how it works among patients with various timings of T790M emergence and treatment.

\section{Materials and Methods}

Advanced EGFR-mutant lung adenocarcinoma patients with positive T790M mutation in tumor were retrospectively enrolled and observed to determine the outcomes of osimertinib treatment. We evaluated the association between patients' characteristics and the efficacy of osimertinib treatment, particularly with respect to the timing of T790M emergence and osimertinib prescription.

\section{Results}

A total of 91 patients were enrolled, including 14 (15.4\%) with primary and 77 (84.6\%) with acquired T790M mutation. The objective response rate and disease control rate were $60.9 \%$ and $85.1 \%$, respectively. The median progression-free survival (PFS) and overall survival were 11.5 months (95\% confidence interval [Cl], 9.0 to 14.0 ) and 30.4 months $(95 \% \mathrm{Cl}$, 11.3 to 49.5 ), respectively. There was no significant difference in response rate and PFS between primary and acquired T790M populations. In the acquired T790M subgroup, patients who received osimertinib after T790M had been confirmed by rebiopsy had a longer PFS than those with intercalated treatments between rebiopsy and osimertinib prescription (14.0 months [95\% Cl, 9.0 to 18.9] vs. 7.2 months [ $95 \% \mathrm{Cl}, 3.7$ to 10.8]; adjusted hazard ratio, 0.48 [95\% $\mathrm{Cl}, 0.24$ to $0.98 ; p=0.043]$ ). Rebiopsy timing did not influence the outcome.

\section{Conclusion}

Osimertinib prescription with intercalated treatment following rebiopsy but not the timing of T790M emergence influenced the treatment outcome. We suggest that it is better to start osimertinib treatment once T790M mutation has been confirmed by biopsy.

\author{
Key words \\ Epidermal growth factor receptor, Lung adenocarcinoma, \\ Osimertinib
}




\section{Introduction}

Epidermal growth factor receptor (EGFR) mutation is one of the most common oncogenic drivers in lung cancer patients [1,2]. Since EGFR-tyrosine kinase inhibitor (TKI) can offer a better efficacy and quality of life [3,4], it has emerged as an important first line therapy in EGFR-mutant non-small cell lung cancer (NSCLC) patients. Although most EGFRmutant NSCLC patients may experience a good response to EGFR-TKI initially, acquired resistance inevitably occurs, which leads to a progression of the disease [5]. The most common mechanism of acquired resistance is a secondary EGFR mutation that involves a substitution of threonine to methionine at codon 790 (T790M) [6].

In our previous study [7], we suggested that the detection of T790M should not be limited at the time of EGFR-TKI progression because it could also be identified among patients experiencing an interval from progression to initial EGFRTKI treatment as well as among those lacking the continuing EGFR-TKI treatment at the time of rebiopsy. Furthermore, a small portion of EGFR-mutant patients were known to harbor primary T790M before EGFR-TKI treatment [2]. Although previous studies suggested that T790M mutation has a "wax and wane" nature [8], little is known about the true dynamics of T790M mutation and how it interferes with patients' outcomes and treatment results.

Osimertinib is a third-generation EGFR-TKI that selectively targets both sensitizing EGFR mutations and T790M resistance mutation. Several clinical trials have shown that it exhibits promising efficacy and has a more favorable adverse events profile for EGFR-mutant patients resistant to prior EGFR-TKI therapy [9-11], and T790M was found to serve as a key predictor of the efficacy. Clinical trials enrolled patients with T790M mutation detected after disease progression on "the last treatment regimen" [9,11]. However, real-world patients may not always have the chance to receive osimertinib treatment immediately after T790M confirmed by rebiopsy. Currently, it remains unclear whether treatment efficacy varies among patients with different intervals between T790M emergence and osimertinib prescription. We conducted this study to evaluate the association between patients' characteristics and the efficacy of osimertinib treatment, with a particular emphasis on both the timing of T790M emergence and osimertinib prescription.

\section{Materials and Methods}

\section{Patients}

We retrospectively analyzed lung cancer patients who were diagnosed and treated with osimertinib at Taichung Veterans General Hospital (TCVGH) between September 2014 and January 2017. To be eligible for participation in the study, patients were required to have pathologically confirmed lung adenocarcinoma, advanced stage (stage IIIB and IV) disease, known sensitive EGFR mutations in treatmentnaïve tumor specimens, positive T790M mutation in tumor tissue (either primary or acquired after EGFR-TKI progression), a history of osimertinib therapy, and complete clinical follow-up data. Patients were excluded if they had lung tumor with doubtful origin, other active malignancies, T790M detected only in plasma ctDNA, incomplete data records, or received other anti-neoplasm therapy during the course of osimertinib treatment (e.g., chemotherapy and immunotherapy).

\section{Data records and response evaluation}

Clinical data for analysis included patients' age, sex, Eastern Cooperative Oncology Group performance status (ECOG PS), tumor stage, smoking status, EGFR mutation status, biopsy condition, and serial treatment history. Brain metastasis status at the time of osimertinib treatment was also recorded. Tumor, node, and metastases (TNM) staging was done according to the seventh edition of the American Joint Committee for Cancer staging system [12]. Unidimensional measurements as defined by Response Evaluation Criteria in Solid Tumors ver. 1.1 were used in this study [13]. The objective of this study was to compare the efficacy of osimertinib in T790M-positive lung adenocarcinoma patients with various treatment and biopsy conditions. Outcome variables included the objective response rate (ORR), disease control rate (DCR), progression-free survival (PFS), and overall survival (OS).

\section{EGFR mutation analysis}

Tumor specimens were collected and procured for EGFR mutation analysis as previously described [14]. The detection method used in this study was matrix-assisted-laser-desorption-ionization time-of-flight mass spectrometry (MALDITOF MS). The detection spectrum of MALDI-TOF MS is summarized in S1 Table. We performed the testing according to the instructions provided by the MassARRAY system (Sequenom, San Diego, CA). With respect to the biochemical reaction, polymerase chain reaction was used to amplify the 
region containing the tyrosine kinase domain of the EGFR exons 18, 19, 20, and 21. A single nucleotide extension was then performed by primers and corresponding detection probes to amplify the region containing each target mutation. After SpectroClean Resin clean up, samples were loaded onto the matrix of SpectroCHIP by Nanodispenser (Matrix) and then analyzed by Bruker Autoflex MALDI-TOF MS. Data were collected and analyzed by Typer4 software (Sequenom). All the tests were performed by ISO15189-certified TR6 Pharmacogenomics Lab, National Research Program for Biopharmaceuticals (NRPB), at the National Center of Excellence for Clinical Trial and Research of National Taiwan University Hospital.

\section{Statistical methods}

With regard to the rebiopsy timing, patients who had received other systemic treatment between the first EGFRTKI progression and rebiopsy were defined as rebiopsy "with interval from first EGFR-TKI progression" and patients receiving rebiopsy at the time of first EGFR-TKI progression were indicated as rebiopsy "at first EGFR-TKI progression." Patients who had continued receiving EGFR-TKIs treatment within 1 month before rebiopsy, were defined as "with EGFR-TKI treatment at rebiopsy." With regard to the treatment timing, patients who had received any other systemic treatment, such as chemotherapy, immunotherapy, and other targeted therapy, between rebiopsy and the prescription of osimertinib were defined as osimertinib treatment "with intercalated treatment" and patients without intercalated treatments between rebiopsy and osimertinib prescription were indicated as osimertinib treatment "after rebiopsy." We also evaluated the interim between rebiopsy and osimertinib treatment ( $<6$ months vs. $\geq 6$ months). Univariate analyses of ORR and DCR were performed using Fisher's exact test. The Kaplan-Meier method was used to estimate PFS and OS. Differences in survival time were analyzed by log-rank test. Logistic regression model and Cox proportional hazard model were used for multivariate analyses of treatment responses and survival outcomes. In the stepwise procedure, the significant levels for entry and removal were 0.05 and 0.10 , respectively. All statistical tests were carried out using SPSS ver. 15.0 (SPSS Inc., Chicago, IL). Two-tailed tests and $\mathrm{p}$-values of $<0.05$ for significance were used.

\section{Ethical statement}

This study was approved by the Institutional Review Board of Taichung Veterans General Hospital (IRB No. CF12019). Written informed consents for genetic testing and clinical data records were obtained from all patients.

\section{Results}

\section{Patient demographics and osimertinib treatment}

The selection flowchart of the study population is shown in Fig. 1. A total of 91 patients with advanced lung adeno-

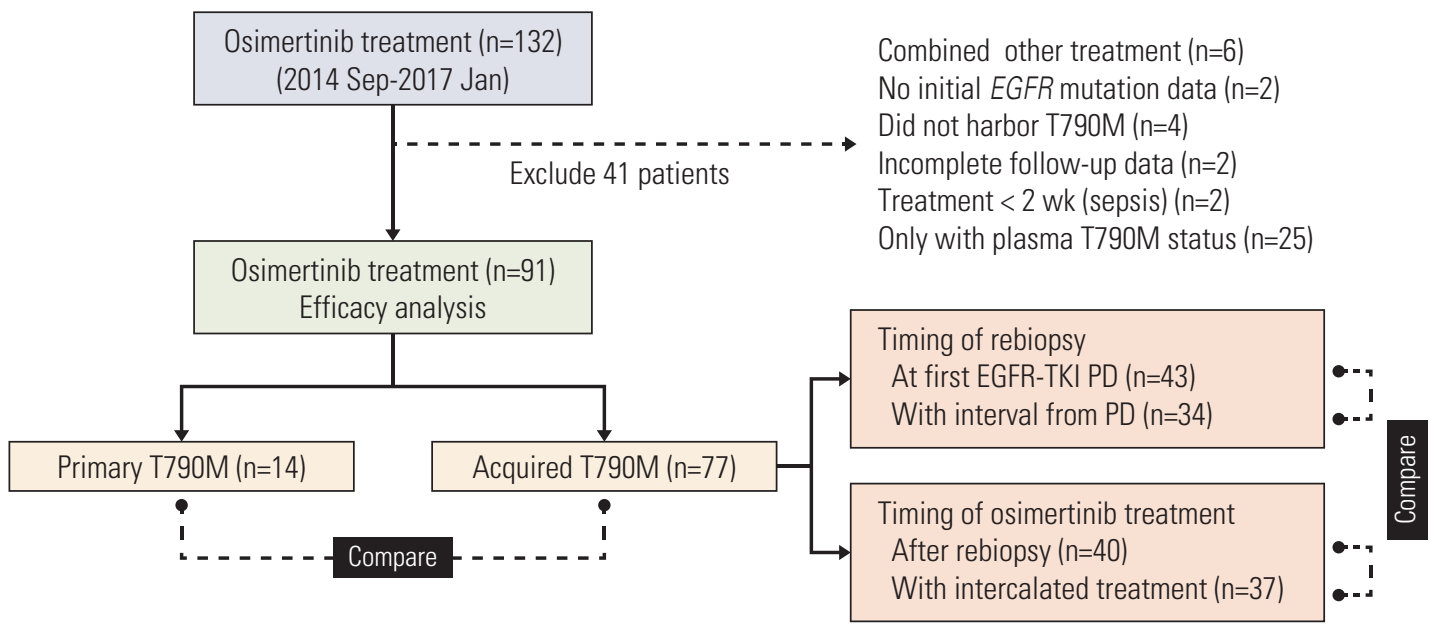

Fig. 1. Patient selection flowchart. EGFR, epidermal growth factor receptor; TKI, tyrosine kinase inhibitor; PD, disease progression. 
Table 1. Univariate analysis of osimertinib best response regarding with patients' demographic data and prior treatment condition $\left(\mathrm{n}=87^{\mathrm{a}}\right)$

\begin{tabular}{|c|c|c|c|c|c|}
\hline Characteristic & No. & ORR $(\%)$ & p-value $e^{b)}$ & DCR $(\%)$ & p-value ${ }^{\text {b) }}$ \\
\hline \multicolumn{6}{|l|}{ Demographic data } \\
\hline \multicolumn{6}{|l|}{ Age (yr) } \\
\hline$<65$ & 49 & 63.3 & 0.662 & 87.8 & 0.547 \\
\hline$\geq 65$ & 38 & 57.9 & & 81.6 & \\
\hline \multicolumn{6}{|l|}{ Sex } \\
\hline Male & 28 & 60.7 & $>0.999$ & 85.7 & $>0.999$ \\
\hline Female & 59 & 61.0 & & 84.7 & \\
\hline \multicolumn{6}{|l|}{ Smoking } \\
\hline Non-smokers & 75 & 58.7 & 0.352 & 85.3 & $>0.999$ \\
\hline Former and current smokers & 12 & 75.0 & & 83.3 & \\
\hline \multicolumn{6}{|l|}{ ECOG PS } \\
\hline $0-1$ & 70 & 65.7 & 0.095 & 91.4 & 0.003 \\
\hline$\geq 2$ & 17 & 41.2 & & 58.8 & \\
\hline \multicolumn{6}{|l|}{ Baseline EGFR mutations } \\
\hline Exon 19 deletions & 47 & 59.6 & 0.385 & 83.0 & 0.661 \\
\hline Exon 21 L858R & 24 & 70.8 & & 83.3 & \\
\hline Others ${ }^{c}$ & 16 & 50.0 & & 93.8 & \\
\hline \multicolumn{6}{|l|}{ Brain metastasis } \\
\hline Yes & 41 & 58.5 & 0.826 & 82.9 & 0.765 \\
\hline No & 46 & 63.0 & & 87.0 & \\
\hline \multicolumn{6}{|l|}{ Prior treatment condition } \\
\hline \multicolumn{6}{|l|}{ First EGFR-TKI regimen ${ }^{\mathrm{d}), e}$} \\
\hline Gefitinib & 37 & 62.2 & 0.844 & 83.8 & $>0.999$ \\
\hline Erlotinib & 42 & 57.1 & & 85.7 & \\
\hline Afatinib & 7 & 71.4 & & 85.7 & \\
\hline \multicolumn{6}{|l|}{ Initial EGFR-TKI treatment ${ }^{\mathrm{d}, e,}$} \\
\hline First line & 70 & 55.7 & 0.088 & 84.3 & $>0.999$ \\
\hline Second line or later & 16 & 81.3 & & 87.5 & \\
\hline \multicolumn{6}{|l|}{ Prior EGFR-TKI(s) treatment ${ }^{\mathrm{d}), e}$ ) } \\
\hline 1 & 59 & 52.5 & 0.081 & 84.7 & 0.428 \\
\hline 2 & 20 & 80.0 & & 90.0 & \\
\hline 3 & 7 & 71.4 & & 71.4 & \\
\hline \multicolumn{6}{|c|}{ Best response to prior EGFR-TKI(s) ${ }^{\mathrm{d}, e)}$} \\
\hline Partial response & 68 & 58.8 & 0.599 & 82.4 & 0.285 \\
\hline Non-responder & 18 & 66.7 & & 94.4 & \\
\hline \multicolumn{6}{|l|}{ PFS of prior EGFR-TKI(s) $(\mathrm{mo})^{\mathrm{d}), e}$} \\
\hline$<12$ & 40 & 57.5 & 0.662 & 80.0 & 0.366 \\
\hline$\geq 12$ & 46 & 63.0 & & 89.1 & \\
\hline \multicolumn{6}{|l|}{ Prior chemotherapy } \\
\hline Naïve & 22 & 54.5 & 0.614 & 90.9 & 0.502 \\
\hline Chemotherapy-treated & 65 & 63.1 & & 83.1 & \\
\hline \multicolumn{6}{|l|}{ EGFR-TKI use before osimertinib ${ }^{f}$} \\
\hline Yes & 50 & 64.0 & 0.514 & 82.0 & 0.544 \\
\hline No & 37 & 56.8 & & 89.2 & \\
\hline \multicolumn{6}{|l|}{ Osimertinib treatment timing $\left.(1)^{g}\right)$} \\
\hline After rebiopsy & 38 & 71.1 & 0.095 & 86.8 & 0.369 \\
\hline With intercalated treatment & 36 & 50.0 & & 77.8 & \\
\hline
\end{tabular}

(Continued to the next page) 
Table 1. Continued

\begin{tabular}{|c|c|c|c|c|c|}
\hline Characteristic & No. & ORR $(\%)$ & p-value ${ }^{\text {b) }}$ & DCR $(\%)$ & p-value $e^{\text {b) }}$ \\
\hline \multicolumn{6}{|c|}{ Osimertinib treatment timing $\left.(2)^{g}\right)$} \\
\hline$<6$ mo from rebiopsy & 60 & 61.7 & 0.769 & 81.7 & $>0.999$ \\
\hline$\geq 6$ mo from rebiopsy & 14 & 57.1 & & 85.7 & \\
\hline
\end{tabular}

ORR, objective response rate; DCR, disease control rate; ECOG PS, Eastern Cooperative Oncology Group performance status; EGFR, epidermal growth factor receptor; TKI, tyrosine kinase inhibitor; PFS, progression-free survival. ${ }^{\text {a) }}$ Exclude 4 patients without measurable lesion, b) By Fisher exact test, ${ }^{\mathrm{c}}$ Include complex mutations involving 19Del or L858R, d)Denote the first and/or second generation EGFR-TKI(s), e) One patient harboring primary T790M did not receive first or second generation

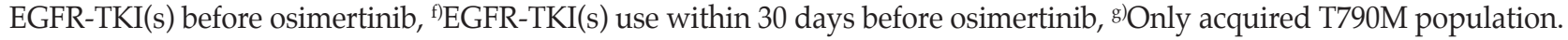

Table 2. Univariate analysis of osimertinib best response regarding with T790M emergence condition $\left(\mathrm{n}=87^{\mathrm{a})}\right)$

\begin{tabular}{|c|c|c|c|c|c|}
\hline Characteristic & No. & ORR $(\%)$ & p-value ${ }^{\text {b) }}$ & $\operatorname{DCR}(\%)$ & p-value ${ }^{\text {b) }}$ \\
\hline \multicolumn{6}{|l|}{ T790M emergence timing } \\
\hline Primary & 13 & 61.5 & $>0.999$ & 100 & 0.202 \\
\hline Acquired & 74 & 60.8 & & 82.4 & \\
\hline \multicolumn{6}{|l|}{ Acquired T790M biopsy timing 1} \\
\hline At first EGFR-TKI PD & 41 & 51.2 & 0.093 & 82.9 & $>0.999$ \\
\hline With interval from PD & 33 & 72.7 & & 81.8 & \\
\hline \multicolumn{6}{|l|}{ Acquired T790M biopsy timing 2} \\
\hline With EGFR-TKI at rebiopsy & 62 & 58.1 & 0.345 & 79.0 & 0.110 \\
\hline Without EGFR-TKI at rebiopsy & 12 & 75.0 & & 100 & \\
\hline \multicolumn{6}{|l|}{ Biopsy location 1} \\
\hline Primary tumor & 29 & 58.6 & 0.818 & 82.8 & 0.753 \\
\hline Metastatic site(s) & 58 & 62.1 & & 86.2 & \\
\hline \multicolumn{6}{|l|}{ Biopsy location 2} \\
\hline Within thorax & 65 & 64.6 & 0.312 & 86.2 & 0.731 \\
\hline Out of thorax & 22 & 50.0 & & 81.8 & \\
\hline
\end{tabular}

ORR, objective response rate; DCR, disease control rate; EGFR, epidermal growth factor receptor; TKI, tyrosine kinase inhibitor; PD, disease progression. ${ }^{a}$ Exclude four patients without measurable target lesion, b) By Fisher exact test, ${ }^{\mathrm{c}}$ EGFR-TKI(s) use within 30 days before rebiopsy or not.

carcinoma harboring T790M and treated with osimertinib were enrolled for outcome analysis. Patients' characteristics are shown in S2 Table. Briefly, the median age was 63 years. Sixty-one patients $(67.0 \%)$ were female and 77 patients $(84.6 \%)$ were non-smokers. Most patients $(79.1 \%)$ received EGFR-TKI as the first line therapy. Sixty-three patients $(69.2 \%)$ received one EGFR-TKI treatment previously and 27 patients $(29.7 \%)$ had received two or more prior EGFR-TKIs. One patient with primary T790M did not receive first or second generation EGFR-TKI before osimertinib treatment. Seventy-three patients $(80.2 \%)$ had ECOG PS 0-1 and 22 patients $(24.2 \%)$ were chemonaïve. Forty-one patients $(45.1 \%)$ had brain metastasis at the time of osimertinib treatment. Exon 19 deletion (19Del) and exon 21 L858R accounted for the most common baseline EGFR mutation types (52.7\% and $27.5 \%$, respectively). Other mutations included one with G719S, one with G719S+S768I, two with 19Del+G719X, and 14 with primary T790M (4 accompanied with 19Del and 10 accompanied with L858R).

After exclusion of four patients without a measurable lesion, the ORR and DCR were $60.9 \%$ and $85.1 \%$, respectively. Survival outcomes were followed up till June 30, 2017. The median PFS and OS were 11.5 months (95\% confidence interval [CI], 9.0 to 14.0) and 30.4 months (95\% CI, 11.3 to $49.5)$, respectively. With regard to the timing of T790M emergence, 14 patients $(15.4 \%)$ harbored primary T790M and 77 patients $(84.6 \%$ ) had acquired T790M after EGFR-TKI treatment. Among the acquired T790M population, rebiopsy was 


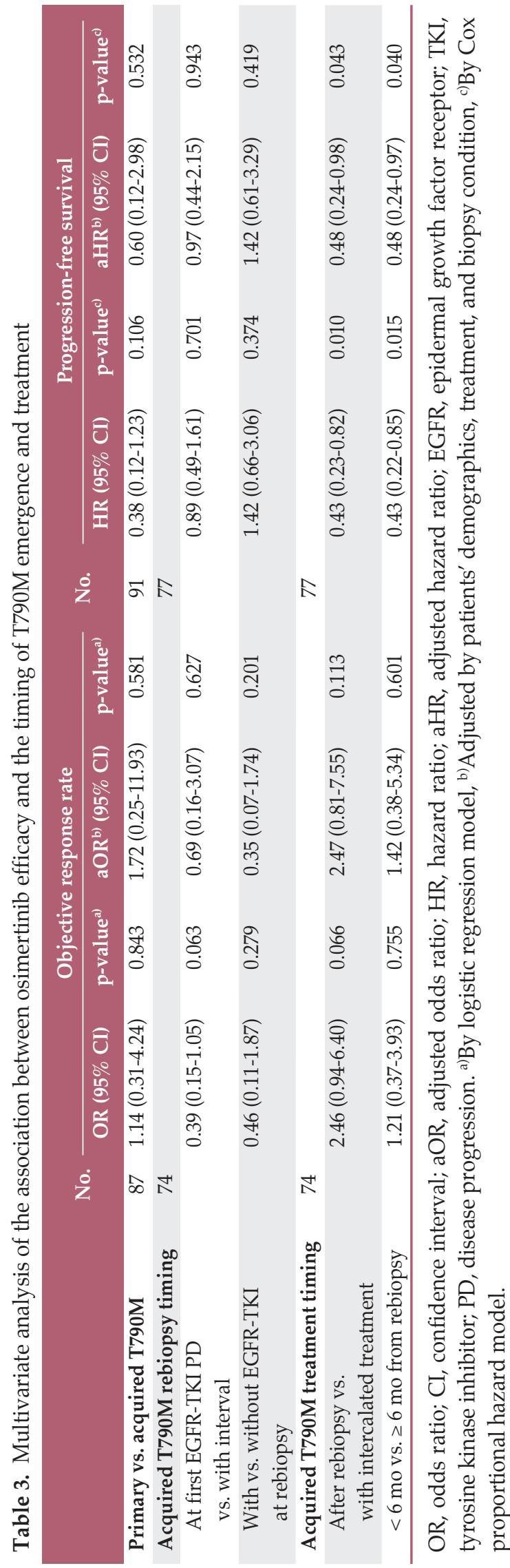

performed at first EGFR-TKI progression in 43 patients and the other 34 patients received rebiopsy following an interval from the first EGFR-TKI progression. In the case of treatment timing, 40 patients received osimertinib after T790M was confirmed by rebiopsy and the other 37 patients had intercalated treatment between rebiopsy and osimertinib prescription.

\section{Osimertinib efficacy and patients' clinical condition}

Univariate analyses of the best response of osimertinib are shown in Tables 1 and 2. In the ORR analysis, no factor correlated significantly with objective response to osimertinib treatment. There were trends of differences in ECOG PS, EGFR-TKI as the first line treatment or not, numbers of prior EGFR-TKI treatments, osimertinib treatment timing, and the rebiopsy timing of acquired T790M. In the DCR analysis, ECOG PS was the only factor that correlated with disease control significantly. Patients with ECOG PS 0-1 were more likely to achieve disease control (91.4\% vs. $58.8 \%, \mathrm{p}=0.003)$.

For the overall population, no covariate reached a significant level in the multivariate logistic regression model for ORR analysis. In the case of DCR analysis, only ECOG PS 0-1 independently correlated with a higher chance of disease control (odds ratio [OR], 9.00; 95\% CI, 2.35 to 34.54; $\mathrm{p}=0.001$ ). In the multivariate Cox proportional hazard model, ECOG 0-1 was the only factor associated with both a longer PFS and OS independently (hazard ratio [HR], 0.32; 95\% CI, 0.16 to $0.63 ; \mathrm{p}=0.001$ and $0.21 ; 95 \% \mathrm{CI}, 0.09$ to $0.49 ; \mathrm{p}<0.001$, respectively).

\section{Osimertinib among patients with primary or acquired T790M}

As shown in Table 2, the ORR and DCR among patients with primary and acquired T790M were $61.5 \%$ vs. $60.8 \%$ and $100 \%$ vs. $82.4 \%$, respectively. Neither p-values were statistically significant. S3 Fig. illustrates the PFS and a trend toward a longer PFS in primary T790M than in acquired T790M population was found (not reached vs. 10.7 months [95\% CI, 8.0 to 13.5], $\mathrm{p}=0.093$ ). Of the primary $\mathrm{T} 790 \mathrm{M}$ patients, 11 were still continuing osimertinib treatment at the time of data cut-off and seven of them experienced more than 10 months' PFS. OS was similar between each group $(\mathrm{p}=0.990)$.

The results of the multivariate analysis of ORR and PFS regarding the timing of T790M emergence are shown in Table 3. There was no significant difference in ORR (adjusted OR, 1.72; 95\% CI, 0.25 to 11.93; $\mathrm{p}=0.581$ ) and PFS (adjusted $\mathrm{HR}, 0.60 ; 95 \% \mathrm{CI}, 0.12$ to $2.98 ; \mathrm{p}=0.532$ ) between each group. 
A
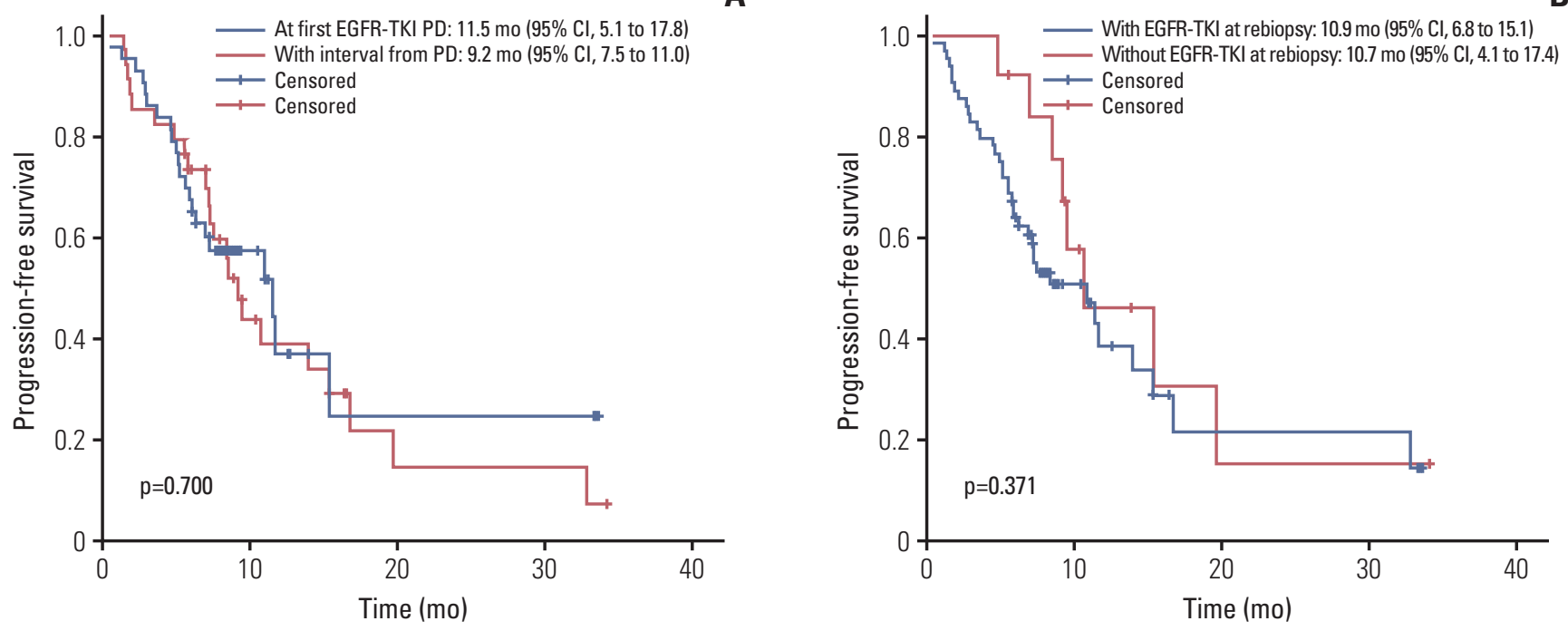

Fig. 2. Kaplan-Meier plot showing progression-free survival of patients with acquired T790M in relation to rebiopsy timing (p-value by log-rank test). EGFR, epidermal growth factor receptor; TKI, tyrosine kinase inhibitor; PD, disease progression; $\mathrm{CI}$, confidence interval.

A
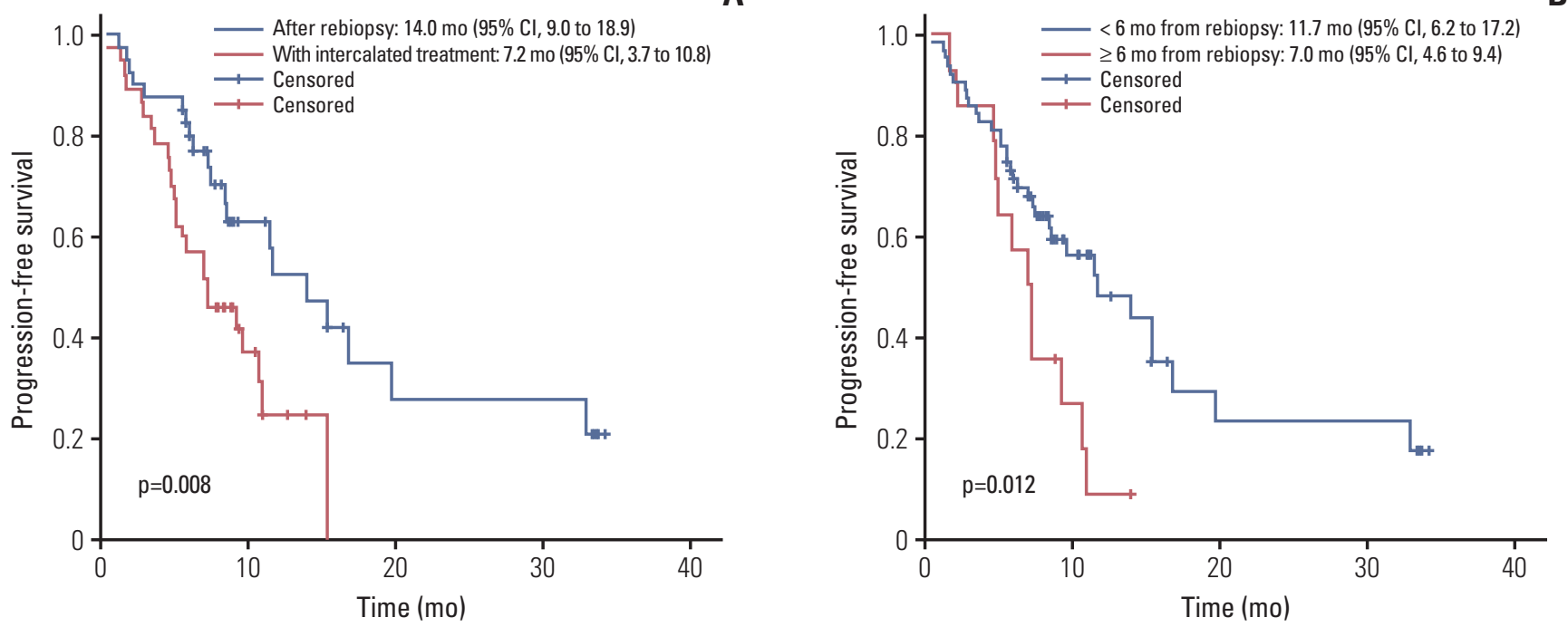

Fig. 3. Kaplan-Meier plot showing progression-free survival of patients with acquired T790M in relation to osimertinib treatment timing (p-value by log-rank test). CI, confidence interval.

\section{Rebiopsy timing and osimertinib efficacy among pati- ents with acquired T790M}

With regard to the rebiopsy timing, we examined the interval between rebiopsy and "first" EGFR-TKI progression because EGFR-mutant patients usually benefit most from the first EGFR-TKI therapy but not the rechallenge [15]. As shown in Table 2, in the acquired T790M subgroup, the ORR and DCR among patients whose T790M detected at first EGFR-TKI progression and after an interval were $51.2 \%$ vs. $72.7 \%$ and $82.9 \%$ vs. $81.8 \%$, respectively. Both p-values were not statistically significant. There were also no significant dif- 
ferences in PFS (11.5 months [95\% CI, 5.1 to 17.8] vs. 9.2 months [95\% CI, 7.5 to 11.0], $\mathrm{p}=0.700$ ) (Fig. 2). Similar results were noted in the analysis of whether patients remained EGFR-TKI at the time of rebiopsy.

In the multivariate analysis (Table 3), there was no significant difference in ORR (adjusted OR, 0.69; 95\% CI, 0.16 to 3.07; $\mathrm{p}=0.627$ ) and PFS (adjusted HR, $0.97 ; 95 \% \mathrm{CI}, 0.44$ to 2.15; $\mathrm{p}=0.943$ ) between patients whose $\mathrm{T} 790 \mathrm{M}$ were detected at the first EGFR-TKI progression and after an interval. Similar results were noted in the analysis of whether patients were kept on EGFR-TKI at the time of rebiopsy.

\section{Treatment timing and osimertinib efficacy among pati- ents with acquired T790M}

In the acquired T790M subgroup as shown in Table 1, the ORR and DCR among patients who received osimertinib after T790M was confirmed by rebiopsy and with intercalated treatment were $71.1 \%$ versus $50.0 \%(\mathrm{p}=0.095)$ and $86.8 \%$ versus $77.8 \%(\mathrm{p}=0.369)$, respectively. Similar results were noted in the analysis of whether patients started osimertinib treatment within 6 months after rebiopsy or not. With regard to the osimertinib treatment timing, there was no significant difference in ORR by the multivariate analysis (Table 3).

In the PFS analysis as shown in Fig. 3, patients who received osimertinib immediately after T790M was confirmed by rebiopsy and who started treatment within 6 months after rebiopsy had a longer survival time (14.0 months; $95 \%$ CI, 9.0 to 18.9 vs. 7.2 months; $95 \%$ CI, 3.7 to 10.8; $\mathrm{p}=0.008$ and 11.7 months; $95 \% \mathrm{CI}, 6.2$ to 17.2 vs. 7.0 months; 95\% CI, 4.6 to $9.4 ; \mathrm{p}=0.012$, respectively). In the multivariate analysis (Table 3), osimertinib treatment started after T790M was confirmed by rebiopsy and within 6 months after rebiopsy significantly correlated with PFS (adjusted HR, 0.48; 95\% CI, 0.24 to $0.98 ; \mathrm{p}=0.043$ and $0.48 ; 95 \% \mathrm{CI}, 0.24$ to 0.97 ; $\mathrm{p}=0.040$, respectively).

\section{Discussion}

The success of EGFR-targeted therapy has led to an era of precision medicine in lung cancer [16]. By now, personalized therapy has moved in the direction of the genotypic evolution of lung cancer, because the most common mechanism of resistance, the T790M mutation, could be overcome by treatment with third generation EGFR-TKI. Among patients who progress after first or second generation EGFR-TKI, T790M is not only a mechanism of resistance, but also serves as an important biomarker of subsequent osimertinib treatment $[10,17]$. The outcome of osimertinib treatment among
T790M-positive patients in our study was comparable with that of previous clinical trials $[9,11]$. However, it should be noted that our cohort consisted entirely of ethnically Asian patients, who were more heavily treated, and there were greater prevalence rates of subjects with brain metastasis and poor performance status. Our results disclosed that ECOG PS remained an important prognostic factor, even in the setting of targeted therapy. Moreover, our findings indicated that the timing of treatment, but not the timing of T790M emergence, may significantly affect the efficacy of osimertinib treatment.

Because previous clinical trials focused on EGFR-TKI-pretreated T790M-positive patients [9-11,17], little is known about the efficacy of osimertinib in patients harboring primary T790M. A study by Hata et al. [18] suggested that T790M could both pre-exist and evolve from the drug-tolerant cells, and that different mechanisms may result in distinct efficacy of treatment. Of them, tumors with pre-exist T790M may be more responsive to third generation EGFR-TKI. In the present study, there were 14 patients with primary T790M. All of them had concomitant sensitizing and T790M mutation in treatment-naïve tumor specimens (4 with 19Del and 10 with L858R). Although the overall efficacy was similar to that of acquired T790M patients, we observed a trend toward a longer PFS in patients with primary T790M ( $p=0.093$ ) and 11 of them were still continuing osimertinib treatment at the time of data cut-off. Hence, we suggest that osimertinib might offer at least similar benefits to patients with primary T790M. A longer follow-up time and prospective studies enrolling more patients are needed to clarify the true efficacy of osimertinib in a primary T790M population.

Since previous studies suggested that primary and acquired T790M mutation had distinct preferences of concomitant mutation partners, different prognostic meanings, and potentially a different pathogenesis [18-21], they may represent two distinct entities. We performed a subgroup analysis of the acquired T790M population. Our previous study suggested that T790M could be identified not only at the time of EGFR-TKI progression or with the continuing EGFR-TKI treatment at the time of rebiopsy, but also in patients with intercalated treatment after EGFR-TKI progression [7]; herein, we further demonstrated that rebiopsy timing did not influence the efficacy of osimertinib. The results were similar with those of AURA and AURA2 studies [9,11], showing that the efficacy of osimertinib was consistent across subgroups with various last treatment regimens, in which the T790M was identified. The aforementioned data suggest that T790M might carry a significant oncogenic activity per se; hence, whenever it is detected, patients could benefit from anti-T790M treatment. For patients without suitable lesions for rebiopsy at the time of EGFR-TKI progression, an attempt to perform rebiopsy should be considered during the subse- 
quent treatment courses [7].

Both AURA and AURA2 studies enrolled patients with positive T790M detected after progression of the last treatment $[9,11]$. ASTRIS (Real World Treatment Study of AZD9291 for Advanced/Metastatic EGFR T790M Mutation NSCLC, NCT02474355) is an ongoing phase III study designed to assess the efficacy and safety of osimertinib in a real world setting [22]. However, only T790M mutation confirmed after last treatment progression is accepted for participation. In the present study, we further analyzed the interval between rebiopsy and osimertinib prescription and found that intercalation of other systemic therapy and length of the interval influenced the outcome of subsequent osimertinib treatment. Although ORR and DCR were not statistically significant in the analysis of treatment timing, patients with intercalated treatments or who started treatment more than 6 months after rebiopsy had a significantly shorter PFS. Maemondo et al. [23] compared the efficacy of gefitinib and chemotherapy for EGFR-mutant NSCLC patients in a randomized phase III study, and demonstrated that the ORR of gefitinib in first line and second line settings were $73.7 \%$ and $58.5 \%$, respectively. Similar results were observed in a phase II study by Sugio et al. [24], and the PFS of the overall population, 7.1 months, was shorter than that observed in a pure treatment-naïve population [16]. Moreover, in the case of ALK mutation, a different efficacy of crizotinib could be observed between treatment-naïve and chemotherapyrefractory populations (PFS, 10.9 months vs. 7.7 months, respectively) $[25,26]$. These results imply that a complicated interaction exists between treatments. Because both the sensitizing EGFR mutation and T790M frequency are dynamic and would be diminished by the effective treatment following EGFR-TKI progression using quantitative method [27], the T790M level at the time of osimertinib prescription might be different in patients with or without intercalated treatment, which could explain at least partly the distinct outcome in this study. The underlying mechanisms of cancer evolution during the path of treatments require further investigation. Herein, we suggest that it is better to start osimertinib treatment once T790M has been confirmed by rebiopsy.

Liquid biopsy using plasma ctDNA, which is more convenient and carries lower risks, is an alternative method to obtain genetic information about tumors [28]. As compared with sensitizing mutations, the detection of T790M in plasma is associated with a lower sensitivity and specificity [29], which resulted in a different outcome of osimertinib treatment. Therefore, we did not include patients whose T790M mutation was only detected in plasma to avoid the potentially confounding variables. Our previous study showed that dynamic plasma EGFR mutation status can serve as an independent outcome predictor of EGFR-TKI therapy [30].
Theoretically, this concept can be applied in T790M detection and osimertinib therapy, too. Better platforms of liquid biopsy are needed to set up for clinical application.

The major limitation of the present study is the retrospective nature of this investigation. Although data were collected retrospectively, we tried to ensure the validity of patients' characteristics, as well as the correlation between treatment course and outcome measurement. Primary T790M accounted for $15.4 \%$ of the study cohort but this did not represent the true prevalence of the whole population [2]. There were only a limited number of available cases involving rare mutations with T790M in our study and in clinical trials $[9,11]$; hence, the efficacy of osimertinib among this subgroup remains unknown. Moreover, further studies are needed to evaluate the dynamics and pathogenesis of T790M mutation during the course of lung cancer treatment.

In conclusion, patients with primary T790M could also benefit from osimertinib treatment and the efficacy was at least similar to that of acquired $7790 \mathrm{M}$. The results of our analysis of the acquired T790M patients suggest that the timing of treatment, but not the timing of rebiopsy, influenced the outcome of osimertinib treatment.

\section{Electronic Supplementary Material}

Supplementary materials are available at Cancer Research and Treatment website (http:// www.e-crt.org).

\section{Conflicts of Interest}

Conflict of interest relevant to this article was not reported.

\section{Acknowledgments}

We would like to thank the Comprehensive Cancer Center of Taichung Veterans General Hospital for assisting with data collection and management and we also thank the NRPB Pharmacogenomics Lab and the NCFPB Integrated Core Facility for Functional Genomics for their technical support. 


\section{References}

1. Cancer Genome Atlas Research Network. Comprehensive molecular profiling of lung adenocarcinoma. Nature. 2014;511: 543-50.

2. Hsu KH, Ho CC, Hsia TC, Tseng JS, Su KY, Wu MF, et al. Identification of five driver gene mutations in patients with treatment-naive lung adenocarcinoma in Taiwan. PLoS One. 2015;10:e0120852.

3. Greenhalgh J, Dwan K, Boland A, Bates V, Vecchio F, Dundar $Y$, et al. First-line treatment of advanced epidermal growth factor receptor (EGFR) mutation positive non-squamous nonsmall cell lung cancer. Cochrane Database Syst Rev. 2016;(5): CD010383.

4. Thongprasert S, Duffield E, Saijo N, Wu YL, Yang JC, Chu DT, et al. Health-related quality-of-life in a randomized phase III first-line study of gefitinib versus carboplatin/paclitaxel in clinically selected patients from Asia with advanced NSCLC (IPASS). J Thorac Oncol. 2011;6:1872-80.

5. Jackman D, Pao W, Riely GJ, Engelman JA, Kris MG, Janne PA, et al. Clinical definition of acquired resistance to epidermal growth factor receptor tyrosine kinase inhibitors in nonsmall-cell lung cancer. J Clin Oncol. 2010;28:357-60.

6. Yu HA, Arcila ME, Rekhtman N, Sima CS, Zakowski MF, Pao $\mathrm{W}$, et al. Analysis of tumor specimens at the time of acquired resistance to EGFR-TKI therapy in 155 patients with EGFRmutant lung cancers. Clin Cancer Res. 2013;19:2240-7.

7. Tseng JS, Su KY, Yang TY, Chen KC, Hsu KH, Chen HY, et al. The emergence of T790M mutation in EGFR-mutant lung adenocarcinoma patients having a history of acquired resistance to EGFR-TKI: focus on rebiopsy timing and long-term existence of T790M. Oncotarget. 2016;7:48059-69.

8. Hata A, Katakami N, Yoshioka H, Kaji R, Masago K, Fujita S, et al. Spatiotemporal T790M heterogeneity in individual patients with EGFR-mutant non-small-cell lung cancer after acquired resistance to EGFR-TKI. J Thorac Oncol. 2015;10: 1553-9.

9. Goss G, Tsai CM, Shepherd FA, Bazhenova L, Lee JS, Chang GC, et al. Osimertinib for pretreated EGFR Thr790Met-positive advanced non-small-cell lung cancer (AURA2): a multicentre, open-label, single-arm, phase 2 study. Lancet Oncol. 2016;17:1643-52

10. Janne PA, Yang JC, Kim DW, Planchard D, Ohe Y, Ramalingam SS, et al. AZD9291 in EGFR inhibitor-resistant nonsmall-cell lung cancer. N Engl J Med. 2015;372:1689-99.

11. Yang JC, Ahn MJ, Kim DW, Ramalingam SS, Sequist LV, Su WC, et al. Osimertinib in pretreated T790M-positive advanced non-small-cell lung cancer: AURA Study phase II extension component. J Clin Oncol. 2017;35:1288-96.

12. Edge SB, Byrd DR, Compton CC, Fritz AG, Greene FL, Trotti AE. AJCC cancer staging handbook. 7th ed. New York: Springer; 2009.

13. Eisenhauer EA, Therasse P, Bogaerts J, Schwartz LH, Sargent D, Ford R, et al. New response evaluation criteria in solid tumours: revised RECIST guideline (version 1.1). Eur J Cancer. 2009;45:228-47.
14. Su KY, Chen HY, Li KC, Kuo ML, Yang JC, Chan WK, et al. Pretreatment epidermal growth factor receptor (EGFR) T790M mutation predicts shorter EGFR tyrosine kinase inhibitor response duration in patients with non-small-cell lung cancer. J Clin Oncol. 2012;30:433-40.

15. Chang GC, Tseng CH, Hsu KH, Yu CJ, Yang CT, Chen KC, et al. Predictive factors for EGFR-tyrosine kinase inhibitor retreatment in patients with EGFR-mutated non-small-cell lung cancer: a multicenter retrospective SEQUENCE study. Lung Cancer. 2017;104:58-64.

16. Mok TS, Wu YL, Thongprasert S, Yang CH, Chu DT, Saijo N, et al. Gefitinib or carboplatin-paclitaxel in pulmonary adenocarcinoma. N Engl J Med. 2009;361:947-57.

17. Mok TS, Wu YL, Ahn MJ, Garassino MC, Kim HR, Ramalingam SS, et al. Osimertinib or platinum-pemetrexed in EGFR T790M-positive lung cancer. N Engl J Med. 2017;376:629-40.

18. Hata AN, Niederst MJ, Archibald HL, Gomez-Caraballo M, Siddiqui FM, Mulvey HE, et al. Tumor cells can follow distinct evolutionary paths to become resistant to epidermal growth factor receptor inhibition. Nat Med. 2016;22:262-9.

19. Chen LY, Molina-Vila MA, Ruan SY, Su KY, Liao WY, Yu KL, et al. Coexistence of EGFR T790M mutation and common activating mutations in pretreatment non-small cell lung cancer: a systematic review and meta-analysis. Lung Cancer. 2016;94:46-53.

20. Liu Y, Sun L, Xiong ZC, Sun X, Zhang SL, Ma JT, et al. Metaanalysis of the impact of de novo and acquired EGFR T790M mutations on the prognosis of patients with non-small cell lung cancer receiving EGFR-TKIs. Onco Targets Ther. 2017;10:2267-79.

21. Matsuo N, Azuma K, Sakai K, Hattori S, Kawahara A, Ishii H, et al. Association of EGFR exon 19 deletion and EGFR-TKI treatment duration with frequency of T790M mutation in EGFR-mutant lung cancer patients. Sci Rep. 2016;6:36458.

22. Real world treatment study of AZD9291 for advanced/ metastatic EGFR T790M mutation NSCLC (ASTRIS) [Internet]. Bethesda, MD: ClinicalTrials.gov; 2015 [cited 2017 Dec 1]. Available from: https://clinicaltrials.gov/ct2/show/NCT02474355/.

23. Maemondo M, Inoue A, Kobayashi K, Sugawara S, Oizumi S, Isobe $\mathrm{H}$, et al. Gefitinib or chemotherapy for non-small-cell lung cancer with mutated EGFR. N Engl J Med. 2010;362: 2380-8.

24. Sugio K, Uramoto H, Onitsuka T, Mizukami M, Ichiki Y, Sugaya $\mathrm{M}$, et al. Prospective phase II study of gefitinib in nonsmall cell lung cancer with epidermal growth factor receptor gene mutations. Lung Cancer. 2009;64:314-8.

25. Shaw AT, Kim DW, Nakagawa K, Seto T, Crino L, Ahn MJ, et al. Crizotinib versus chemotherapy in advanced ALK-positive lung cancer. N Engl J Med. 2013;368:2385-94.

26. Solomon BJ, Mok T, Kim DW, Wu YL, Nakagawa K, Mekhail $\mathrm{T}$, et al. First-line crizotinib versus chemotherapy in ALK-positive lung cancer. N Engl J Med. 2014;371:2167-77.

27. Zheng D, Ye X, Zhang MZ, Sun Y, Wang JY, Ni J, et al. Plasma 
EGFR T790M ctDNA status is associated with clinical outcome in advanced NSCLC patients with acquired EGFR-TKI resistance. Sci Rep. 2016;6:20913.

28. Siravegna G, Marsoni S, Siena S, Bardelli A. Integrating liquid biopsies into the management of cancer. Nat Rev Clin Oncol. 2017;14:531-48.

29. Oxnard GR, Thress KS, Alden RS, Lawrance R, Paweletz CP, Cantarini $\mathrm{M}$, et al. Association between plasma genotyping and outcomes of treatment with osimertinib (AZD9291) in advanced non-small-cell lung cancer. J Clin Oncol. 2016;34: 3375-82.

30. Tseng JS, Yang TY, Tsai CR, Chen KC, Hsu KH, Tsai MH, et al. Dynamic plasma EGFR mutation status as a predictor of EGFR-TKI efficacy in patients with EGFR-mutant lung adenocarcinoma. J Thorac Oncol. 2015;10:603-10. 